\title{
Research on the Time-Domain Measurement Method of Low- Frequency Splitting for Hemispherical Resonator
}

\author{
Shaoliang Li $\left(\mathbb{D},{ }^{1}\right.$ Hao Yang, ${ }^{1}$ Wanliang Zhao $\mathbb{D},{ }^{1,2}$ Rui Qu, ${ }^{3}$ Jie Duan, ${ }^{1}$ Yijie Rong, ${ }^{1}$ Xin Jin, ${ }^{3}$ \\ and Chaojiang $\mathbf{L i}^{3}$ \\ ${ }^{1}$ Shanghai Aerospace Control Technology Institute, Shanghai 201109, China \\ ${ }^{2}$ School of Mechanical Engineering, Nanjing University of Science and Technology, Nanjing 210094, China \\ ${ }^{3}$ School of Mechanical Engineering, Beijing Institute of Technology, Beijing 100081, China \\ Correspondence should be addressed to Wanliang Zhao; zhaodada999@163.com
}

Received 3 February 2021; Revised 19 February 2021; Accepted 7 April 2021; Published 7 May 2021

Academic Editor: Vasilios N. Katsikis

Copyright ( 2021 Shaoliang Li et al. This is an open access article distributed under the Creative Commons Attribution License, which permits unrestricted use, distribution, and reproduction in any medium, provided the original work is properly cited.

\begin{abstract}
The measurement of resonator's frequency splitting is a critical issue in vibratory gyroscopes, which would be elaborately treated in practical applications. The high-precision measurement of frequency splitting plays a significant role in frequency tuning control. A novel time-domain method of frequency splitting measurement for hemispherical resonator based on the standing wave swing effect was proposed. The frequency splitting value of the resonator can be directly obtained by taking the reciprocal of the one cycle time of standing wave swings, rather than through the frequency difference between two resonant modes. To begin with, the method was analyzed theoretically, and the measurement resolution and accuracy of the method were researched in detail. Simulation and experimental results showed that the frequency splitting value can be effectively obtained by measuring the period of the standing wave swings, improving the fine measurement resolution and high accuracy. The frequency splitting of lower than $0.007 \mathrm{~Hz}$ has to be effectively obtained in the experiment. It is found that the measurement error is a small proportional part of frequency splitting value, so the measurement accuracy is very high when the frequency splitting is very low. Therefore, this time-domain method would contribute to the measurement of ultralow-frequency splitting for high-Q resonators.
\end{abstract}

\section{Introduction}

Hemispherical resonant gyroscope (HRG) is a kind of vibrating gyroscope without high-speed rotor and movable parts, which is in operation based on the Coriolis effect. HRG offers many advantages which includes long running time, high accuracy, small structure, and short start-up time [1]. Therefore, it has a wide range of application prospects in highprecision and long-life inertial navigation such as aviation systems, precision guide missile, and other fields [2]. Due to the imperfect fabrication process, machining errors and manufacturing defects will inevitably occur on the resonator, which results in uneven circumferential distribution of mass, rigidity, and $Q$ factor [3]. These problems are reflected in the vibration process of the harmonic oscillator, which will lead to the phenomenon of frequency splitting. Facts have proved that the primary error source of HRG is the drift caused by the frequency splitting [4-6]. The key issue in vibratory gyroscopes, therefore, will be the measurement of resonator's frequency splitting, which would be elaborately treated by mechanical balancing [7] or electrical treatment [8-10] in practical applications. When the $Q$ factor is high (typically up to $10^{7}$ ) and the frequency splitting is very low, it is a great challenge to obtain the measurement, because the frequency splitting value is sufficiently low to reduce the gyro drift.

Choi and Kim [11] estimated the frequency splitting amount of the natural frequencies based on the mathematical model of imperfect shell with multiple point masses. But no specific measurement method is proposed. Matveyev [7] proposed a method for determining the azimuth and frequency splitting of the inherent stiffness axis based on the amplitude frequency characteristics and phase angle characteristics. The resonator is excited by a sweeping voltage signal, and the vibration signal is recorded by two capacitive sensors at an 
angle of $\pm 22.5^{\circ}$. The excitation voltage sweeps in steps of $0.001 \mathrm{~Hz}$. The frequency splitting could be measured by analyzing the voltage amplitude and phase shift of the two capacitive sensors. This method has high measurement reliability and good stability which is wildly used in MEMS hemispherical resonator gyros [12-15] and MEMS gyros [16-19]. However, the disadvantage of this approach is that two precision capacitors need to be built around the resonator which is a challenging work to ensure the measurement accuracy. Besides of that, the measurement accuracy is limited by the step of the sweeping voltage. Cho et al. [20] mounted the resonator on a stacked PZT actuator and applied $z$-axis linear acceleration to the resonator. All the modes can be actuated due to the structural asymmetry in the resonator. And the resonant mode shapes and frequency spectrum are verified with a Polytec PSV 400 scanning laser vibrometer. Wang et al. [21] determined the resonant frequency through a PSV-500 and frequency-sweeping process. The frequency split of cylindrical axisymmetric-shell resonators was estimated based on FEM simulations and measured by getting the two natural frequencies based on resonator's amplitude frequency response (AFR) characteristic [22, 23].

Measuring each resonant mode's frequency separately was the main methods have been previously used. The resolution of frequency splitting suffers from the limitations of the $Q$ factor of the resonators and the scanning step of exciting force. The measurement method of frequency splitting for a high- $Q$ hemispherical resonator based on a standing wave swing effect was simply discussed previously [24].

In this paper, the time-domain measurement method of low-frequency splitting for a hemispherical resonator is demonstrated and analyzed in detail, which differs from our previous work in the analysis in detail and the further research of the measurement resolution and accuracy by the comparisons with the traditional AFR method and FFT analysis. A complete set of theoretical analysis and simulations of the time-domain measurement method based on the standing wave swing effect was investigated. The measurement resolution and accuracy of this method is researched by comparative experiments with the AFR method and FFT analysis. The experimental results show that the measurement method has a high-frequency resolution and accuracy. And the method is very suitable for the measurement of low frequency splitting for high- $Q$ resonators.

\section{Theoretical Analysis and Simulation}

2.1. Theoretical Analysis. An ideal hemispherical resonator has a completely axisymmetric structure (as shown in Figure $1(\mathrm{a}))$, so the two working vibration mode $(n=2)$ are degenerated and the natural frequencies are exactly the same, as shown in Figure 1(b).

Due to the processing shape and position deviations of the hemispherical resonator, such as circularity and coaxiality, and the anisotropy of the circumferential density and Young's modulus of the resonator material, the two modes of the harmonic oscillator will develop into two $45^{\circ}$ natural axes, the nature frequencies corresponding to the secondorder bending modes of the two different natural axes reach the maximum and minimum, respectively (as shown in Figure 2). The difference between the two natural frequencies is called frequency splitting. And the two natural frequency axes are called natural stiffness axis. If the excitation of the harmonic oscillator is not along the natural axis direction, the frequency splitting will cause the standing wave of the resonator mode to slowly drift toward the natural axis until the vibration is along the natural axis direction, resulting in a gyro drift.

The two-dimensional dynamic model of an ideal axisymmetric hemispherical resonator could be presented as

$$
\begin{aligned}
& \ddot{x}+\omega x-2 k \Omega \dot{y}=0, \\
& \ddot{y}+\omega y+2 k \Omega \dot{x}=0,
\end{aligned}
$$

where $x$ and $y$ are the displacements of two vibration modes, $\omega$ is the natural frequency of the vibration mode, $k$ is the precession factor $(k=0.277$ for the $n=2$ mode of hemispherical resonator), and $\Omega$ is the external angular velocity. For ideal axisymmetric hemispherical resonator, the natural frequency of two $n=2$ modes is completely the same. When the external angular velocity is ignored, the vibration of two modes would be independently harmonic vibrations. The relationship of amplitudes and phases is denoted as

$$
\begin{aligned}
& x=x_{0} \cos (\omega t+\alpha), \\
& y=y_{0} \cos (\omega t+\beta),
\end{aligned}
$$

where $x_{0}, y_{0}$ is the initial amplitude and $\alpha, \beta$ is the initial phase.

Considering the damping issue of the vibration, the twodimensional dynamic model of the hemispherical resonator can be expressed as

$$
\begin{aligned}
& \ddot{x}+\frac{\omega}{Q} \dot{x}+\omega^{2} x-4 k \Omega \dot{y}=0, \\
& \ddot{y}+\frac{\omega}{Q} \dot{y}+\omega^{2} y-4 k \Omega \dot{x}=0,
\end{aligned}
$$

where $Q$ is the $Q$ factor of the damping vibration. And the vibration of two modes is as follows:

$$
\begin{aligned}
& x=x_{0} e^{-(\omega / 2 Q) t} \cos (\omega t+\alpha), \\
& y=y_{0} e^{-(\omega / 2 Q) t} \cos (\omega t+\beta) .
\end{aligned}
$$

When the external angular velocity is ignored, the vibration of two modes would be exponentially decayed.

For an incompletely axisymmetric hemispherical resonator, the two $n=2$ vibration modes of the resonator are not degenerate. And the natural frequencies of the two modes are not equal due to the frequency splitting. The simplified dynamic model of the hemispherical resonator could be expressed by the following formula: 


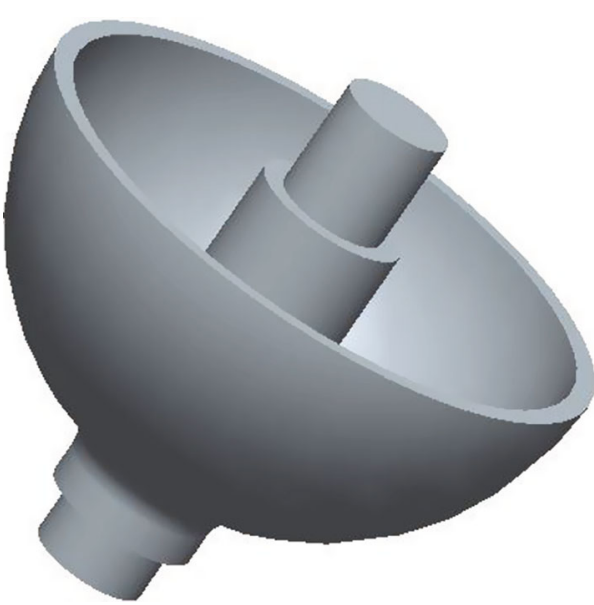

(a)

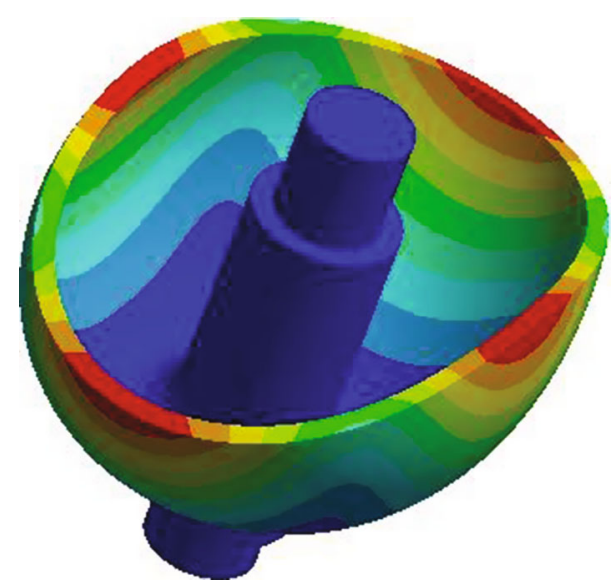

(b)

FIgURE 1: Basic schematic of the hemispherical resonator and vibration mode. (a) Typical structure. (b) $n=2$ vibration mode.

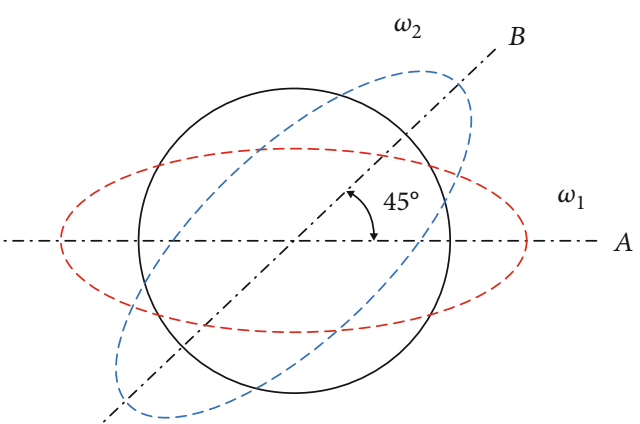

FIgURE 2: Schematic of the vibration mode of hemispherical resonator.

$$
\begin{aligned}
& \ddot{x}+\frac{\omega_{0}}{Q} \dot{x}+\omega_{1}^{2} x-4 k \Omega \dot{y}=0, \\
& \ddot{y}+\frac{\omega_{0}}{Q} \dot{y}+\omega_{2}^{2} y+4 k \Omega \dot{x}=0,
\end{aligned}
$$

where $\omega_{1}$ and $\omega_{2}$ are the resonant frequencies of two vibration modes, respectively; $\omega_{0}=\left(\omega_{1}+\omega_{2}\right) / 2$ when the $Q$ factor inconsistency of the two modes is ignored. The external angular velocity is also negligible when analyzing the frequency splitting. Therefore, the vibration of two modes is as follows:

$$
\begin{aligned}
& x=x_{0} e^{-\left(\omega_{0} / 2 Q\right) t} \cos \omega_{1} t, \\
& y=y_{0} e^{-\left(\omega_{0} / 2 Q\right) t} \cos \omega_{2} t .
\end{aligned}
$$

Thus, the vibration at the azimuth $\varphi$ of hemispherical resonator can be represented as

$$
\begin{aligned}
w(\varphi, t)= & x \cos 2 \varphi+y \sin 2 \varphi=x_{0} \cos 2 \varphi \mathrm{e}^{-\left(\omega_{0} / 2 Q\right) t} \cos \omega_{1} t \\
& +y_{0} \sin 2 \varphi \mathrm{e}^{-\left(\omega_{0} / 2 Q\right) t} \cos \omega_{2} t .
\end{aligned}
$$

2.1.1. Analyze the Vibration on the Effect of Frequency Splitting. It can be seen from the formula above, the $Q$ factor could be ignored when analyzing the vibration on the effect of frequency splitting.

The $n=2$ vibration mode of the resonator is excited along by a certain azimuth through the specific excitation method. As a result, the description of resonator's initial vibration is shown.

$$
w(\varphi, t)=A \cos 2\left(\varphi-\varphi_{0}\right) \cos \omega t
$$

where $A$ is the initial vibrating amplitude, $\varphi_{0}$ is the azimuth between the standing wave and the normal axis, and $\varphi$ is the azimuth of the standing wave.

According to Matveev's analysis, standing waves would be destroyed over time under the influence of frequency splitting. And the resonator's vibration could be expressed as the superposition of two orthogonal vibration waves, which is

$w(\varphi, t)=A \cos 2 \varphi \cos 2 \varphi_{0} \cos \omega_{1} t+A \sin 2 \varphi \sin 2 \varphi_{0} \cos \omega_{2} t$

where $\omega_{1}$ and $\omega_{2}$ are the resonant frequencies of two vibration modes, respectively. The frequency splitting value of hemispherical resonator is defined as

$$
\Delta f=\frac{1}{2 \pi}\left(\Delta_{1}+\Delta_{2}\right)=\frac{1}{2 \pi}\left(\omega_{2}-\omega_{1}\right)
$$

where $\omega\left(\omega_{1}<\omega<\omega_{2}\right)$ is a reference frequency; $\Delta_{1}$ $\left(\Delta_{1}=\omega-\omega_{1}\right)$ and $\Delta_{2}\left(\Delta_{1}=\omega_{2}-\omega\right)$ are two offset frequencies.

And the resonator's vibration is rewritten as

$$
\begin{aligned}
w(\varphi, t)= & A \cos 2 \varphi \cos 2 \varphi_{0} \cos \Delta_{1} t \cos \omega t \\
& +A \sin 2 \varphi \sin 2 \varphi_{0} \cos \Delta_{2} t \cos \omega t \\
& +A \cos 2 \varphi \cos 2 \varphi_{0} \cos \Delta_{1} t \sin \omega t \\
& -A \sin 2 \varphi \sin 2 \varphi_{0} \cos \Delta_{2} t \sin \omega t
\end{aligned}
$$


It can be derived that the vibrating energy at the position $\varphi$ on resonator can be described as

$$
\begin{aligned}
E(\varphi, t)= & A^{2} \cos ^{2} 2 \varphi_{0} \cos ^{2} 2 \varphi \cos ^{2} \Delta_{1} t+A^{2} \sin ^{2} 2 \varphi_{0} \sin ^{2} 2 \varphi \cos ^{2} \Delta_{2} t \\
& +2 A^{2} \cos 2 \varphi_{0} \cos 2 \varphi \sin 2 \varphi_{0} \sin 2 \varphi \cos \Delta_{1} t \cos \Delta_{2} t \\
& +A^{2} \cos ^{2} 2 \varphi_{0} \cos ^{2} 2 \varphi \sin ^{2} \Delta_{1} t+A^{2} \sin ^{2} 2 \varphi_{0} \sin ^{2} 2 \varphi \sin ^{2} \Delta_{2} t \\
& -2 A^{2} \cos 2 \varphi_{0} \cos 2 \varphi \sin 2 \varphi_{0} \sin 2 \varphi \sin \Delta_{1} t \sin \Delta_{2} t \\
= & A^{2} \cos ^{2} 2 \varphi_{0} \cos ^{2} 2 \varphi+A^{2} \sin ^{2} 2 \varphi_{0} \sin ^{2} 2 \varphi \\
& +2 A^{2} \cos 2 \varphi_{0} \cos 2 \varphi \sin 2 \varphi_{0} \sin 2 \varphi \cos (2 \pi \Delta \mathrm{ft}) .
\end{aligned}
$$

It can be obtained from (12) that the energy of a certain position on resonator is demodulated by the frequency splitting value. And the demodulation period time is

$$
T=\frac{2 \pi}{2 \pi \Delta f}=\frac{1}{\Delta f} .
$$

Thus, the frequency splitting of the hemispherical resonator can be calculated by measuring the period time of the resonator's vibrating energy.

Take the partial differential of the vibrating energy, which is

$\frac{\partial E(\varphi, t)}{\partial \varphi}=-2 A^{2} \cos 4 \varphi_{0} \sin 4 \varphi+2 A^{2} \sin 4 \varphi_{0} \cos 4 \varphi \cos (2 \pi \Delta f t)$.

The azimuth of standing wave is presented as

$$
\tan 4 \varphi=\tan 4 \varphi_{0} \cos (2 \pi \Delta f) t .
$$

It can be concluded from (15) that the azimuth of standing wave is demodulated by the frequency splitting value which results in wave swings under the influence of frequency splitting.

2.1.2. The Analysis of Effect of $Q$ Factor on Frequency Splitting Measurement. Without external interference, the vibration of hemispherical resonator tends to ring down freely through the impact of vibration damping. It implies that the $Q$ factor could affect the frequency splitting measurement.

Formula (9) could be reexpressed as the following formula under the influence of $Q$ factor.

$w(\varphi, t)=A_{0} \mathrm{e}^{-\left(\omega_{0} / 2 Q\right) t}\left(\cos 2 \varphi \cos 2 \varphi_{0} \cos \omega_{1} t+\sin 2 \varphi \sin 2 \varphi_{0} \cos \omega_{2} t\right)$.

From (16), the vibration would decay completely due to damping. The standing wave swing effect is rarely observed if the vibration attenuated to zero during the measurement process. The frequency splitting is hardly settled consequently. Thereby, the resolution of the frequency splitting requires that the vibration cannot be attenuated to the amplitude which is too small to be detected during the period time (T) shown in formula (13).
Formula (12) is represented as

$$
\begin{aligned}
E(\varphi, t)= & A^{2} \mathrm{e}^{-\left(\omega_{0} / \mathrm{Q}\right) t}\left[\cos ^{2} 2 \varphi_{0} \cos ^{2} 2 \varphi+\sin ^{2} 2 \varphi_{0} \sin ^{2} 2 \varphi\right. \\
& \left.+2 \cos 2 \varphi_{0} \cos 2 \varphi \sin 2 \varphi_{0} \sin 2 \varphi \cos (2 \pi \Delta \mathrm{ft})\right] .
\end{aligned}
$$

From the formula above, the frequency splitting is much more accessible when the stand wave swing amplitude reaches a maximum. In this azimuth of the hemispherical resonator, the expression for vibration energy is

$$
E(t)=A^{2} \mathrm{e}^{-\left(\omega_{0} / \mathrm{Q}\right) t}\{1+\cos (2 \pi \Delta \mathrm{ft})\} .
$$

Let the initial time be zero, and the time will be

$$
2 \pi \Delta \mathrm{ft}_{\mathrm{i}}=2 i \pi+\pi, i=0,1,2 \cdots,
$$

where $t_{i}$ is defined as the situation when the vibration energy is one minimum value during the vibration. The formula above can be rewritten as

$$
t_{i}=\frac{2 i+1}{2 \Delta f} .
$$

The detection of effective vibration is limited by the resolution of the detection devices. It will be barely detected when the vibration signal is less than the noise of device.

The resolution of the detection device is recorded as $e_{k}$ which is characterized by the ratio of the minimum vibration amplitude to the full range of the detection device. The vibration reaches to the full scale of the detection devices at time $t_{0}$ . In order to distinguish one period time of the standing wave swing, it requires that

$$
\frac{E\left(t_{1}\right)}{E\left(t_{1}\right)}=\frac{e^{-\left(\omega_{0} / Q\right) t_{1}}}{e^{-\left(\omega_{0} / Q\right) t_{0}}} \geq\left(e_{k}\right)^{2}
$$

which can be reduced to

$$
\Delta f=-\frac{\omega_{0}}{2 \mathrm{Q} \ln e_{k}} .
$$

One conclusion can be drawn that under a certain resolution of the vibration detection, the resolution of frequency splitting is higher as the $Q$ factor increasing. The measurement method is very suitable for the high- $Q$ resonators, because the resolution of frequency splitting is very high when $Q$ factor is high enough.

2.2. Simulations. The time-domain measurement method of frequency splitting based on the standing wave swing effect is simulated in MATLAB based on the above analysis. In the simulations, the natural frequency of the resonator is $5000 \mathrm{~Hz}$. The initial vibration amplitude is $5 \mu \mathrm{m}$. The frequency splitting value of the resonator is $0.015 \mathrm{~Hz}$. And the $Q$ factor is 1500000 . The simulation results are shown in Figure 3. Lines 1 and 4 are the vibration attenuation curves when the standing wave coincident with stiffness principal 


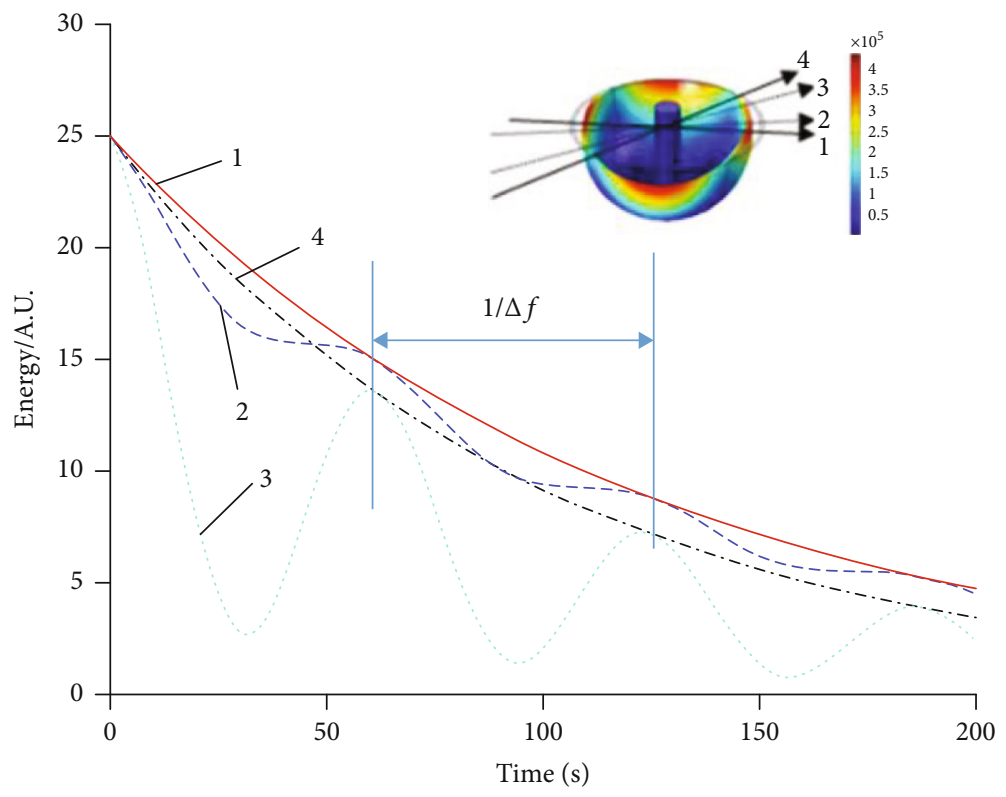

FIGURE 3: Simulation results of the vibrating energy.

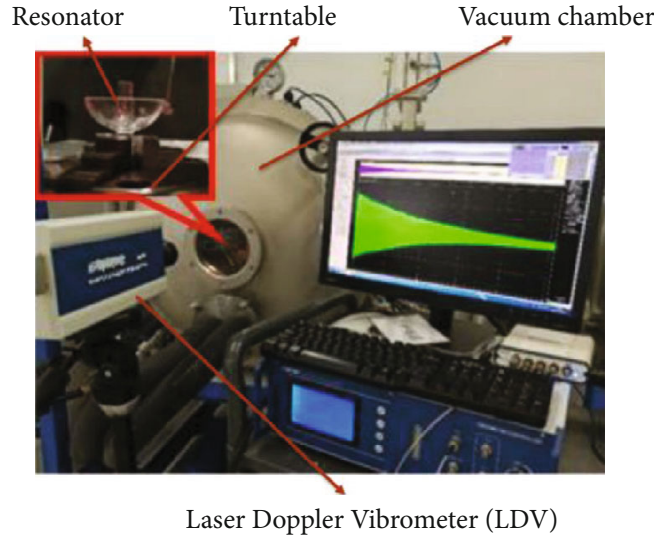

Figure 4: Picture of experimental setup.

axis. Lines 2 and 3 are the vibration attenuation curves when the standing wave does not coincident with stiffness principal axis. It shows that a periodic oscillation signal can be obtained when the exciting and measuring direction is in the middle of two principal axes. And the frequency splitting value could be calculated by taking the reciprocal value of the signal's period time.

2.3. Experimental Setup and Procedure. An experimental apparatus based on a laser Doppler vibrometer (LDV) is set up (as shown in Figure 4) in order to measure the frequency splitting of resonators based on the standing wave swing effect.

2.3.1. Experimental Setup. As shown in Figure 4, the hemispherical resonator is fixed on a turntable which could rotate within the range of $0^{\circ} \sim 360^{\circ}$ in a vacuum chamber. The initial vibration of hemispherical resonator is excited by a striking hammer in a vacuum chamber. And the vibration information is recorded by a LDV.

2.3.2. Experimental Procedure. As a first step, adjust the exciting and measuring directions of the hemispherical resonator. Typically, the exciting point, measuring point, and center of resonator are in the same axial line for simplicity.

Evacuate the vacuum chamber to a certain pressure. The resonator's $Q$ factor varies with the pressure of the vacuum chamber. In the experiment, the pressure in the chamber should be smaller than $10^{-5} \mathrm{~Pa}$ to make sure the resonator get higher $Q$ factor.

Strike the resonator in any initial orientation (set as $0^{\circ}$ ) to excite the initial vibration by the striking hammer in a vacuum chamber which is controlled by a pulse switch outside the chamber. Then, record the vibration signal of the resonator through a LDV.

The vibration signals are processed by filtering and fitting to get the energy exchange period time induced by the standing wave swing effect. The frequency splitting value could be obtained by taking the reciprocal value of the period time $(T)$. As a matter of fact, the two natural frequencies and splitting value could also be obtained by the FFT processing the vibration signals.

\section{Results and Discussions}

In this section, the resonator's frequency splitting is measured based on the method mentioned above. And the measurement resolution and accuracy of the method emphatically analyzed in detail. At last, this method is compared with the traditional AFR method and the FFT analysis.

3.1. The Measurement Results of Hemispherical Resonator's Frequency Splitting. The frequency splitting of two typical 


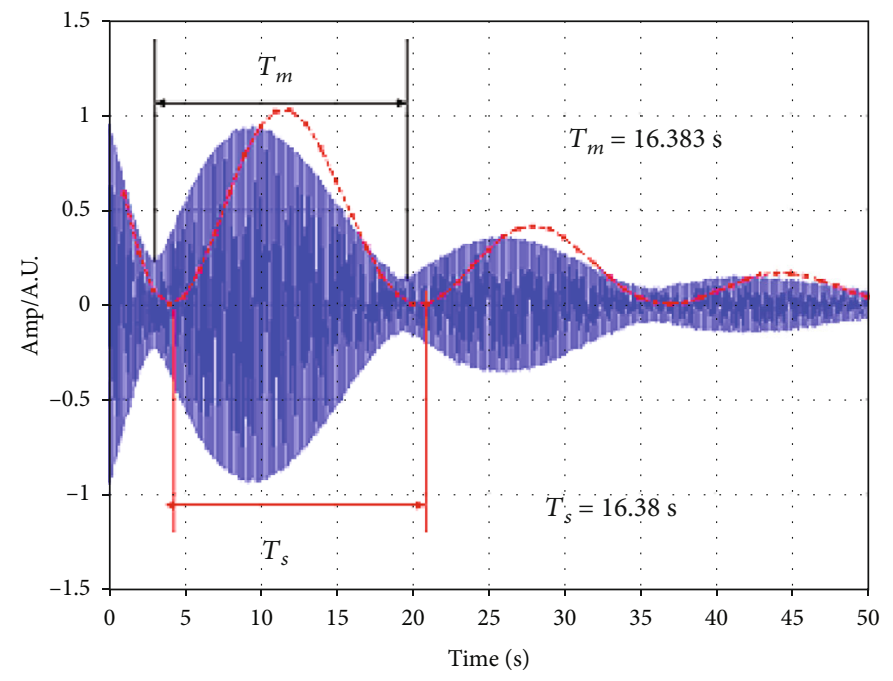

(a)

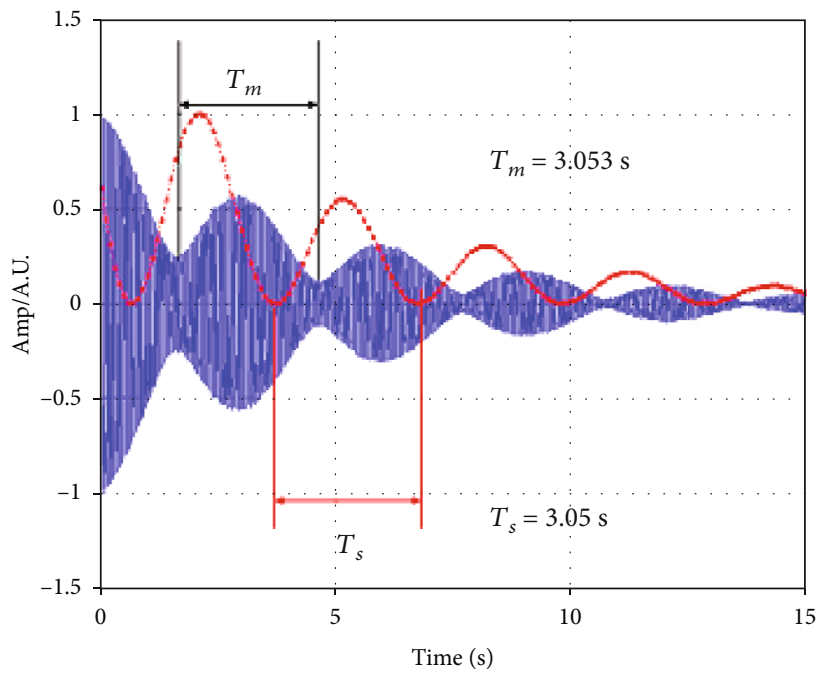

(b)

FIGURE 5: The comparison between simulation and experimental results. (a) 1\# resonator. (b) 2\# resonator.

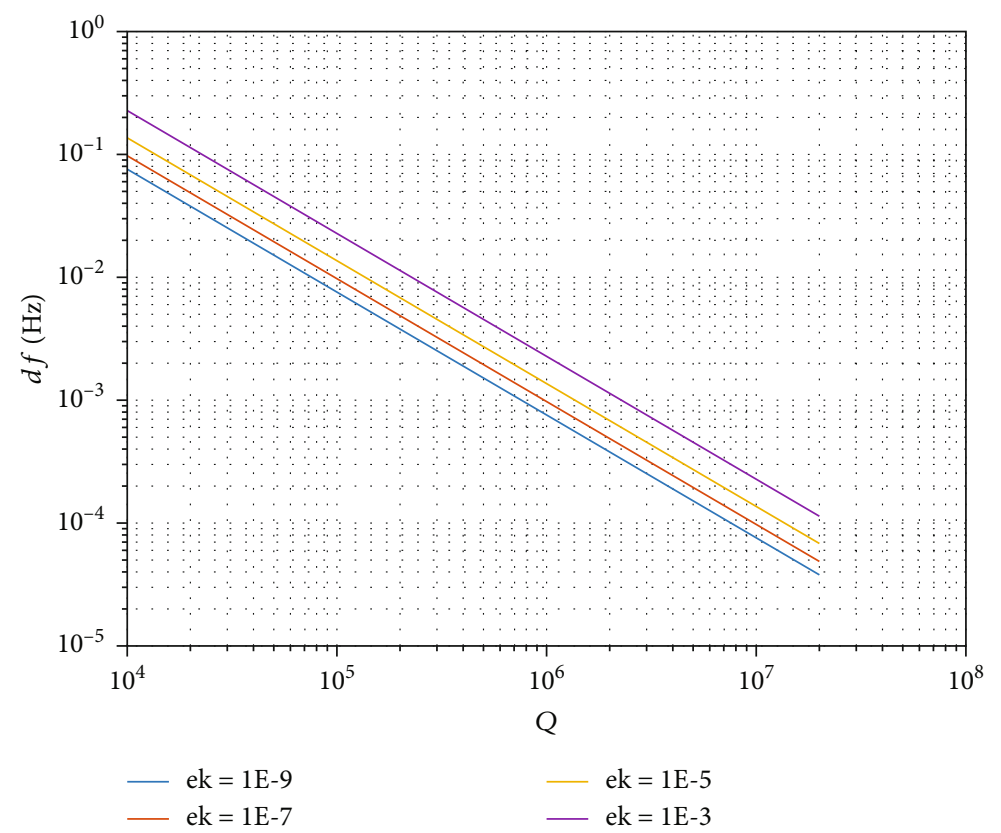

FIgURE 6: The simulation results of the measurement resolution.

hemispherical resonators is measured using the method mentioned above. As reported in ref. [24], it is validated that the frequency splitting, as well as the $Q$ factor of the resonator could be obtained effectively by the measurement method. The results show that the frequency splitting value of $1 \#$ resonator is $0.0610 \mathrm{~Hz}$ and the $Q$ factor is 410000 . And the frequency splitting value of $2 \#$ resonator is $0.3275 \mathrm{~Hz}$ and the $Q$ factor is 98000 .

The presented method is confirmed by comparing the simulation results and the experimental results. The parameters obtained in the experiment $(Q$, frequency, $\Delta f)$ are substituted into the simulation model to obtain the simulation results under the resonator's real parameters. And the comparison results are shown in Figure 5. The blue lines in the figure are the measured curves of the $1 \#$ and 2\# resonators, and the red lines are the simulated curves under the parameters of measured data. It can be seen from the comparison that the simulation results are consistent with the experimental results. The difference phase between the two curves is caused by the selection of starting point in experimental data. The period time of vibrating signal between the simulation and experimental results is consistent which corroborate the correctness of this method.

3.2. The Measurement Resolution Analysis. According to the analysis of effect of $Q$ factor on frequency splitting 

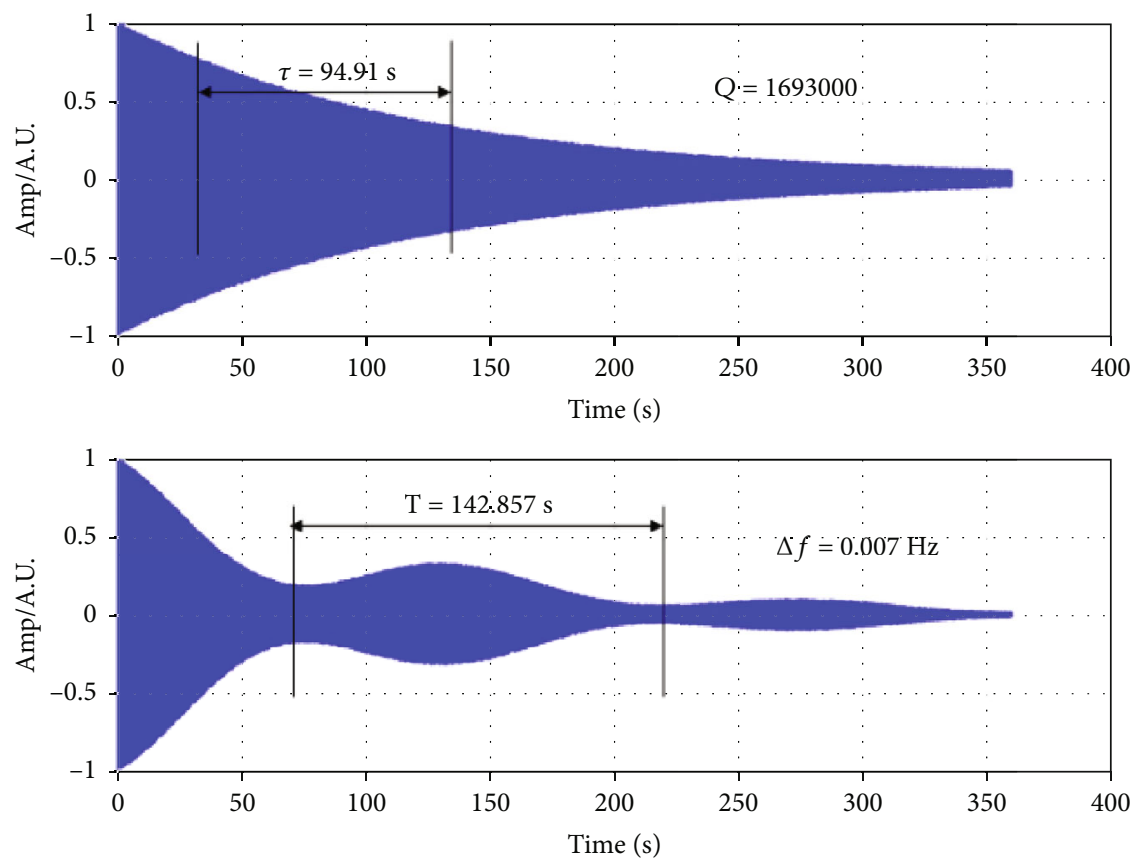

Figure 7: Experimental results of the resonator with lower frequency splitting.

TABLE 1: Experimental results of the resonator.

\begin{tabular}{lcccc}
\hline No. & $T_{1}(\mathrm{~s})$ & $T_{2}(\mathrm{~s})$ & Period time $(\mathrm{s})$ & $\triangle f(\mathrm{~Hz})$ \\
\hline \multirow{3}{*}{$1 \#$} & 2.967 & 19.350 & 16.383 & 0.0610 \\
& 19.350 & 35.740 & 16.390 & 0.0610 \\
& 35.740 & $52.390 /^{*}$ & 16.650 & 0.0601 \\
& 1.603 & 4.656 & 3.053 & 0.3275 \\
$2 \#$ & 4.656 & 7.707 & 3.051 & 0.3278 \\
& 7.707 & 10.750 & 3.043 & 0.3286 \\
& 10.750 & 13.810 & 3.060 & 0.3268 \\
\hline
\end{tabular}

*The vibration signal is too small to detect; therefore, it induces errors.

measurement and formula (22), it can be obtained that the frequency splitting measurement resolution based on the standing wave swing effect depends on the $Q$ factor of the resonator and the limit resolution of the detection devices. The higher the $Q$ value of the resonator and the higher the resolution of the detection devices, the higher the resolution of the frequency splitting measurement is of this method. It is simulated based on formula (22).

The simulation result is shown in Figure 6. The natural frequency of resonator is set to $5000 \mathrm{~Hz}$. In the paper, the resolution of the detection device (LDV) is about $1 \mathrm{E}(-5)$ as shown by the orange line. Therefore, it is verified by measuring a resonator with lower frequency splitting as shown in Figure 7. The experimental results show that the frequency splitting of $0.007 \mathrm{~Hz}$ could be easily obtained while the $Q$ factor is more than 1.69 million. The result is basically consistent with the simulation results in Figure 6. Therefore, the resolution of frequency splitting value could reach to the level of $10^{-4} \mathrm{~Hz}$ if the $Q$ factor is more than 10 million.
3.3. The Measurement Error Analysis. The measurement method based on the standing wave swing effect is essentially converting measuring the natural frequency of each mode into a period time based on the resonator's inherent characteristics. As can be seen from the experimental results, the measurement error of this method is mainly on account of the reading error at the measurement time. The vibrating period time can be expressed by

$$
T_{m}=T+k T k \mid<<1,
$$

where $T$ is the real period time without errors and $k$ is a small proportion estimation error. Therefore, the measured frequency splitting value is

$$
\begin{aligned}
& \Delta f_{m}=\frac{1}{T_{m}}=\left(1-k+k^{2}+\cdots\right) \Delta f, \\
& \Delta f_{m} \approx \Delta f+k \Delta f,
\end{aligned}
$$

where $\Delta f$ is the real frequency splitting value of the resonator without errors.

It can be seen that the measurement error is a small proportion of the frequency splitting value. The smaller the frequency splitting, the higher the measurement accuracy is. In this paper, the frequency splitting value is calculated by selecting different swing period of $1 \#$ and $2 \#$ resonators as shown in Figure 5. The result is shown in Table 1. For 1\# resonator, the period time of the third period has a little deviation compared with the first two periods, because the vibration signal has been greatly decayed and it is too small to distinguish which results in extra errors. In the measurement process, the first few periods with large amplitude 


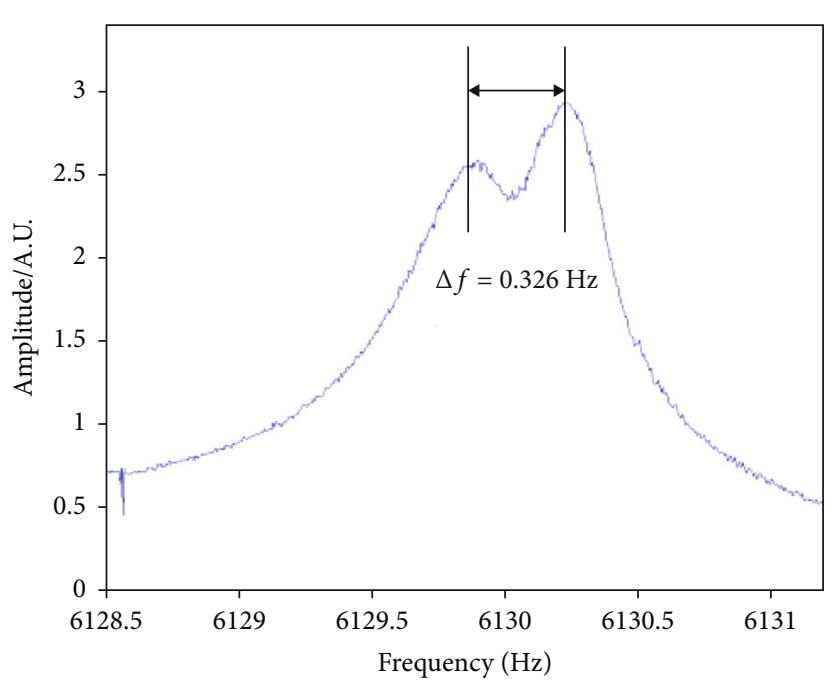

(a)

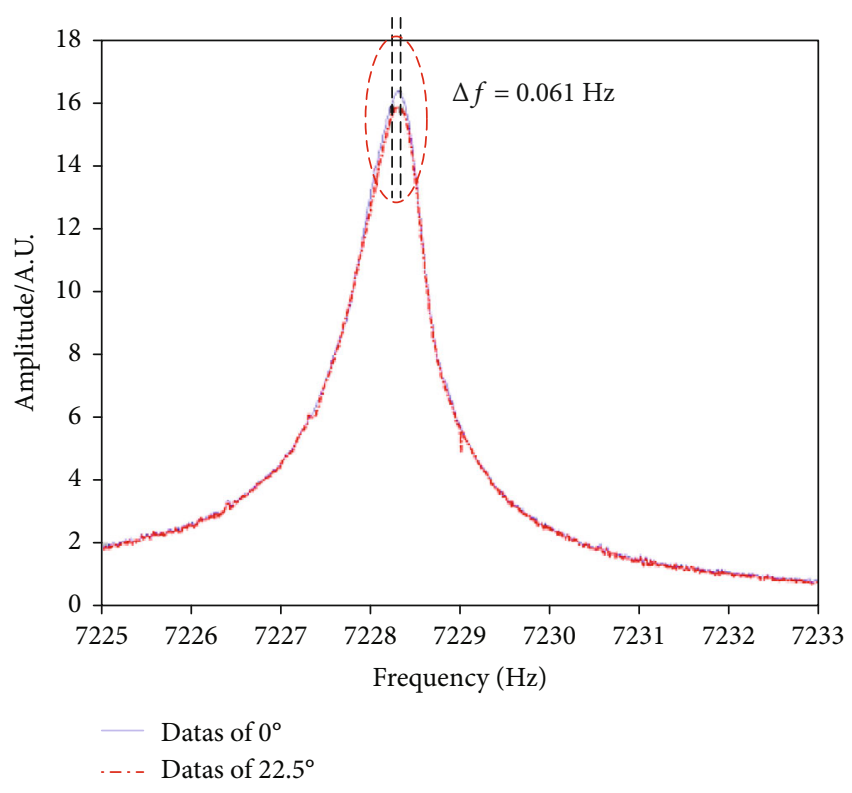

(b)

Figure 8: The measurement results of frequency splitting base on AFR. (a) Resonator 2\#. (b) Resonator 1\#.

should be selected to avoid extra errors. Conclusion reached from the results that the lower the frequency splitting value, the lower the measurement error is. Therefore, this method presented is particularly suitable for the measurement of ultra-low frequency splitting for high- $Q$ hemispherical resonators.

\subsection{Comparison with AFR Method and FFT Analysis}

3.4.1. AFR Method. As reported, the measurement method of frequency splitting based on AFR is widely used in many areas. However, its measurement accuracy is not enough owing to the resolution of the frequency is limited by the sweeping step of hardware. In the paper, the frequency splitting of hemispherical resonators $1 \#$ and $2 \#$ was measured based on AFR method for comparison, by the experimental setup reported in the previous article [25]. The hemispherical resonator is excited by a piezoelectric exciter in the sweeping frequency range of the resonator's natural frequency. The sweeping frequency step is $0.002 \mathrm{~Hz}$. The amplitude frequency response curves of the resonator in the orientation of $0^{\circ}$ and $22.5^{\circ}$ are obtained, respectively.

The experimental results are shown in Figure 8. For resonator 2\#, the two resonant peaks are easily distinguished in the case of $0.326 \mathrm{~Hz}$ frequency splitting value. However, for resonator $1 \#$, the two resonant peaks are hardly distinguished in the case of the frequency splitting value lower than $0.061 \mathrm{~Hz}$. Although the measurement resolution of AFR would be a little better when the resonator's $Q$ factor is higher, a sweep frequency exciter should be placed inside the vacuum chamber, which made the setup more complicated and expensive. It would be still limited by the sweeping step of hardware and the sweeping process. In addition, the natural frequency is vulnerable by the impact of temperature changing which also make poor measurement repeatability base on AFR method. Therefore, this measurement method presented is more suitable for lower frequency splitting for high- $Q$ resonators, while the previous method based on AFR is more suitable for larger frequency splitting.

3.4.2. FFT Analysis of the Vibration Signal. The vibration signal of the resonator could also be analyzed through fast Fourier transform (FFT), as shown in Figure 9. The results show that the natural frequencies of each mode and the frequency splitting value could be obtained through the FFT analysis of the vibration signal. However, the natural frequency peak of the FFT signal would be flattened which could result in measuring errors. There are two main reasons for that. To begin with, the frequency resolution is limited by the amount of the data for FFT analysis. And the natural frequency could be in the interval of two distinguishable frequencies. Moreover, the natural frequencies of vibrating modes fluctuate depend on the external environment during the process of the ringdown vibration which would lead to the broadening of the FFT frequency spectrum. For 1\# resonator, as shown in Figure 9(a), the natural frequency of one mode is in the range of $7228.1069 \mathrm{~Hz}-7228.1128 \mathrm{~Hz}$; and the natural frequency of the other mode is $7228.1680 \mathrm{~Hz}$ (the peak not flattened). Therefore, the frequency splitting value of $1 \#$ resonator is in the range of $0.0552 \mathrm{~Hz}-0.0611 \mathrm{~Hz}$, while the measured frequency splitting value is $0.0610 \mathrm{~Hz}$ through time-domain analysis. For the $2 \#$ resonator, as shown in Figure 9(b), the natural frequency of one mode is in the range of $6129.8584 \mathrm{~Hz}-6129.8828 \mathrm{~Hz}$; and the natural frequency of the other mode is $6130.2002 \mathrm{~Hz}-6130.2246 \mathrm{~Hz}$. Therefore, the frequency splitting value of $2 \#$ resonator is in the range of $0.3174 \mathrm{~Hz}-0.3662 \mathrm{~Hz}$, while the measured frequency splitting value is in the range of $0.3268 \mathrm{~Hz}-0.3278 \mathrm{~Hz}$ through time-domain analysis. The experimental results 


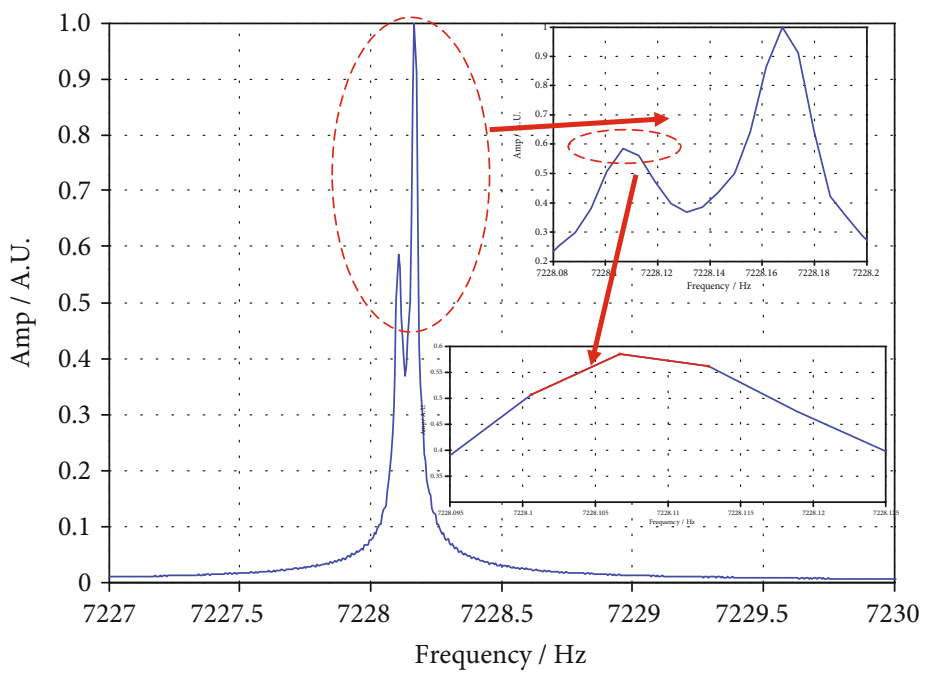

(a)

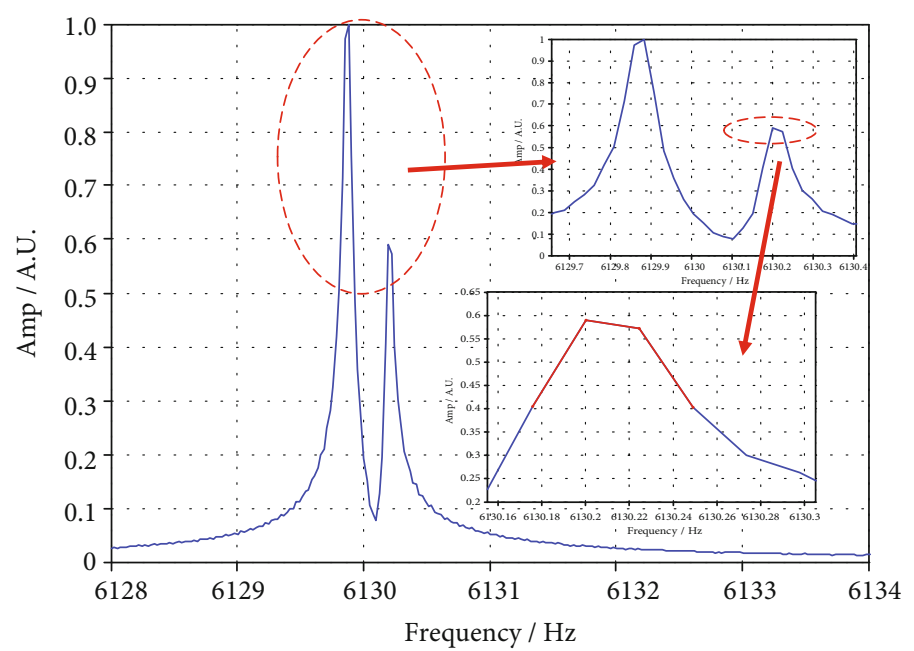

(b)

FIGURE 9: The FFT analysis results of vibrating signals. (a) 1\# resonator. (b) 2\# resonator.

show that, in the normal case of large frequency splitting value measurement, it could be obtained through the FFT analysis of the vibration signal. While in the case of the ultralow-frequency splitting measurement, the method presented in this paper has higher measuring accuracy by the time domain analysis.

\section{Conclusions}

The time-domain measurement method for low-frequency splitting of hemispherical resonators is researched in detail in the paper. The frequency splitting value of the resonator can be directly obtained without calculating the frequency difference between the two resonance modes. The experimental results reveal that the proposed method can effectively obtain a frequency lower than $0.007 \mathrm{~Hz}$. And based on the proven evaluation, the resolution of frequency splitting value could reach to the level of $10^{-4} \mathrm{~Hz}$ if the $Q$ factor is more than 10 million. Since the measurement error only makes up only a small fraction of the frequency splitting value, the measurement accuracy is much higher than the AFR method and FFT analysis. The time-domain method can not only greatly improve the measurement resolution of frequency splitting but also simplify the measurement procedure. Consequently, the measurement method holds great promise prospect in the field of high-precision inertial sensors.

\section{Data Availability}

The datasets used in the experiments and discussed in the paper will be available if requited.

\section{Conflicts of Interest}

The authors declare that they have no conflicts of interest. 


\section{Acknowledgments}

This work was supported by the National Key Research and Development Program of China (Grant No. 2017YFB1104700) and the Program of Shanghai Academic/Technology Research Leader under Project (Grant No. 18XD1421700).

\section{References}

[1] A. Jeanroy, A. Bouvet, and G. Remillieux, "HRG and marine applications," Gyroscope and Navigation, vol. 5, no. 2, pp. 67-74, 2014.

[2] X. Wang, W. Wu, Z. Fang, B. Luo, Y. Li, and Q. Jiang, “Temperature drift compensation for hemispherical resonator gyro based on natural frequency," Sensors, vol. 12, no. 5, pp. 6434-6446, 2012.

[3] P. Pai, F. Chowdhury, C. Mastrangelo, and M. Tabib-Azar, "MEMS-based hemispherical resonator gyroscopes," in SENSORS, 2012 IEEE, pp. 1-4, Taipei, Taiwan, 2012.

[4] V. Zhuravlev, "On the drift of a hemispherical resonator gyro on a rotating base during the quadrature control in fast-time and slow-time modes," Mechanics, vol. 38, no. 3, pp. 8-11, 2003.

[5] Z. Qi, J. Wu, C. Li, W. Zhao, and Y. Cheng, "Micromachined rate-integrating gyroscopes: concept, asymmetry error sources and phenomena," Symmetry, vol. 12, no. 5, p. 801, 2020.

[6] D. D. Lynch, "MRIG frequency mismatch and quadrature control," in 2014 International Symposium on Inertial Sensors and Systems (ISISS), pp. 1-4, Laguna Beach, CA, USA, 2014.

[7] V. A. Matveev, Solid State Wave Gyroscope, National Defence Industry Press, Beijing, 2009.

[8] C. Li, H. Wen, S. Wisher et al., "An FPGA-based Interface system for high-frequency bulk-acoustic-wave microgyroscopes with in-run automatic mode-matching," IEEE Transactions on Instrumentation and Measurement, vol. 69, no. 4, pp. 1783-1793, 2020.

[9] R. Zhang, T. Shao, W. Zhao, A. Li, and B. Xu, "Sliding mode control of MEMS gyroscopes using composite learning," Neurocomputing, vol. 275, pp. 2555-2564, 2018.

[10] Y. Xia, Y. Qi, and X. Cai, "Method to reduce angular increment error of hemispherical resonators unit by time management," Fligth Control and Detect, vol. 1, pp. 41-46, 2018.

[11] S. Y. Choi and J. H. Kim, "Natural frequency split estimation for inextensional vibration of imperfect hemispherical shell," Journal of Sound and Vibration, vol. 330, no. 9, pp. 20942106, 2011.

[12] D. Senkal, M. J. Ahamed, A. A. Trusov, and A. M. Shkel, "Achieving sub-Hz frequency symmetry in micro-glassblown wineglass resonators," Journal of Microelectromechanical Systems, vol. 23, no. 1, pp. 30-38.

[13] D. Senkal, M. J. Ahamed, A. A. Trusov, and A. M. Shkel, "Electrostatic and mechanical characterization of 3-D microwineglass resonators," Sensors and Actuators A: Physical, vol. 215, pp. 150-154, 2014.

[14] J. Bernstein, M. Bancu, E. Cook, M. V. Chaparala, W. A. Teynor, and M. S. Weinberg, "A MEMS diamond hemispherical resonator," Journal of Micromechanics and Microengineering, vol. 23, no. 12, p. 125007, 2013.

[15] C. He, Q. Zhao, Q. Huang et al., "A MEMS vibratory gyroscope with real-time mode-matching and robust control for the sense mode," Sensors Journal, vol. 15, no. 4, pp. 2069-2077, 2014.

[16] D. D. Shin, C. H. Ahn, Y. Chen et al., "Electrostatic tuning of temperature coefficient of frequency of anisotropic diskshaped resonators," in 2017 IEEE International Symposium on Inertial Sensors and Systems (INERTIAL), pp. 164-167, Kauai, HI, USA, 2017.

[17] B. R. Johnson, C. Boynton, E. Cabuz et al., "Toroidal resonators with small frequency mismatch for rate integrating gyroscopes," in 2014 International Symposium on Inertial Sensors and Systems (ISISS), pp. 1-4, Laguna Beach, CA, USA, 2014.

[18] B. Hamelin, A. Daruwalla, and F. Ayazi, "Stiffness trimming of high Q MEMS resonators by excimer laser annealing of germanium thin film on silicon," in 2016 IEEE 29th International Conference on Micro Electro Mechanical Systems (MEMS), pp. 1026-1029, Shanghai, China, 2016.

[19] P. Shao, V. Tavassoli, C. S. Liu, L. Sorenson, and F. Ayazi, "Electrical characterization of ald-coated silicon dioxide micro-hemispherical shell resonators," in 2014 IEEE 27th International Conference on Micro Electro Mechanical Systems (MEMS), pp. 612-615, San Francisco, CA, USA, 2014.

[20] J. Y. Cho, J. Yan, J. A. Gregory, H. W. Eberhart, R. L. Peterson, and K. Najafi, "3-Dimensional blow torch-molding of fused silica microstructures," Journal of Microelectromechanical Systems, vol. 22, no. 6, pp. 1276-1284, 2013.

[21] Y. Wang, Y. Pan, T. Qu, Y. Jia, K. Yang, and H. Luo, "Decreasing frequency splits of hemispherical resonators by chemical etching," Sensors, vol. 18, no. 11, p. 3772, 2018.

[22] Z. Lin, M. Fu, Z. Deng, N. Liu, and H. Liu, "Frequency split elimination method for a solid-state vibratory angular rate gyro with an imperfect axisymmetric-shell resonator," Sensors, vol. 15, no. 2, pp. 3204-3223, 2015.

[23] Z. Su, M. Fu, Q. Li, N. Liu, and H. Liu, "Research on bellshaped vibratory angular rate gyro's character of resonator," Sensors, vol. 13, no. 4, pp. 4724-4741, 2013.

[24] S. Li, Y. Rong, W. Zhao, H. Yang, J. Duan, and S. Zhao, "Measurement method of frequency splitting for high-Q hemispherical resonator based on standing wave swing effect," in 2020 IEEE International Symposium on Inertial Sensors and Systems (INERTIAL), pp. 1-4, Hiroshima, Japan, 2020.

[25] S. L. Li, H. Yang, and Y. Xia, "Measurement method of hemispherical resonator frequency splitting and normal-mode axis azimuth based on amplitude frequency response characteristics," Fligth Control and Detect, vol. 3, pp. 69-74, 2020. 